\title{
Adsorption of Cadmium from Aqueous Solutions Using Low cost Materials-A Review
}

\author{
M.Padmaja $^{1^{*}}$, R.Bhavani ${ }^{2}$, R.Pamila ${ }^{3}$ \\ ${ }^{1,2}$ Department of Civil Engineering, Jawaharlal Nehru Technological University, Anantapuramu, Andhrapradesh, India. \\ ${ }^{3}$ Department of Civil Engineering, Sri Sairam Engineering College, Chennai, Tamilnadu, India. \\ *Corresponding author Email: padmajamegham@gmail.com
}

\begin{abstract}
With the onset of industrialization, a lot of anthropogenic sources of cadmium, including industrial emissions, application of fertilizers and sewage sludge to farm land has lead to the contamination of water bodies, and has increased cadmium uptake by agricultural crops, grown for human consumption. Cadmium when present, even at low concentrations may pose serious health as well as environmental hazards. The use of various materials has been widely investigated as a replacement of recent expensive methods for removing cadmium from water and wastewater. Plant based natural materials, agricultural products, nano materials and industrial wastes are efficiently used as low-cost adsorbents. Until now, most of the researchers have attempted to review the literature for multiple heavy metals. In the current review, an elaborate list of literature has been compiled to provide information on a wide range of natural as well as modified adsorbent materials for the removal of Cadmium from wastewater.
\end{abstract}

Keywords: Adsorbent, agricultural products, cadmium, industrial wastes.

\section{Introduction}

Effluents from many industrial activities are the sources of cadmium into the aqueous environment. Cadmium is an essential element for living organisms, but at increased concentrations it is injurious (Purkayastha et al., 2014).Many conventional methods for the removal of Cadmium such as chemical precipitation (Khosravi and Alamdari 2009), floatation (Rubio et al., 2002), ion exchange (Kang et al., 2004), membrane separation techniques(Al-Rashdi et al., 2013), electrodialysis (Mohammadi et., al 2005), solvent extraction (Mauri et al., 2001) etc; have certain limitations like generation of toxic sludge or other wastes, incomplete metal removal, high reagent and energy requirements. Most of these methods are often ineffective, have complexity in their processes or uneconomical when the concentration of cadmium is higher (10-100 ppm) than the permissible concentration. Adsorption has advantages over the other methods because of simple design with a sludge free environment, have high metal binding capacity, can work over a wide range of $\mathrm{pH}$ and can involve low investment in term of both initial cost and land required (Viraraghavan and Dronamraju, 1993).

\section{Adsorbents}

\subsection{Plant Based Adsorbents}

A wide range of plant based adsorbents are studied for decontaminating the cadmium containing industrial wastes. Batch experiments were conducted at a pH 6 with polyalthia longifolia Seeds as biosorbent (Rao and Rehman, 2012), the sterculia lychnophera seeds were eluted and easily digested with concentrated $\mathrm{HNO}_{3}$ (Liu et., al 2006), Loquat leaves, Loquat ash and moringa oleifera showed a good adsorption at pH 6 \& pH 7 (Al-Dujaili et al., 2012), ( Reddy et al., 2012), acacia leucocephala bark powder used as a sorbent gave an optimum adsorption at $\mathrm{pH} 5$ (Munagapati et al., 2010). Modified sawdust (cedrus deodar wood), azolla filiculoides, wheat bran and coirpith activated carbon are used either as raw or in modified forms (Memon et al., 2007, Ganji et al., 2005, Ozer and Pirinc 2006 and Kadirvelu et al., 2003) were showed in table 1.

Table 1: Adsorption capacity $(\mathrm{mg} / \mathrm{g})$ of plant biomass based materials

\begin{tabular}{|c|c|c|}
\hline Adsorbent & $\begin{array}{c}\text { Adsorption } \\
\text { capacity }(\mathrm{mg} / \mathrm{g})\end{array}$ & References \\
\hline $\begin{array}{l}\text { Polyalthia longifolia Seeds } \\
\text { (Seeds of Indian Mast Tree) }\end{array}$ & 20.74 & $\begin{array}{l}\text { Rao and Rehman } \\
\text { (2012) }\end{array}$ \\
\hline Sterculia lychnophera seeds & 17.5 & Liu Y et al.(2006) \\
\hline $\begin{array}{l}\text { Loquat leaves (Eriobotrya } \\
\text { japonica) }\end{array}$ & 29.24 & $\begin{array}{l}\text { Al-Dujaili et al. } \\
\text { (2012) }\end{array}$ \\
\hline $\begin{array}{l}\text { Loquat ash (Eriobotrya } \\
\text { japonica) }\end{array}$ & 21.32 & $\begin{array}{l}\text { Al-Dujaili et al. } \\
(2012)\end{array}$ \\
\hline Moringa oleifera (CAMOL) & 171.37 & $\begin{array}{l}\text { Reddy et al. } \\
\text { (2012) }\end{array}$ \\
\hline $\begin{array}{l}\text { Acacia leucocephala bark } \\
\text { powder }\end{array}$ & 167.7 & $\begin{array}{l}\text { Munagapati et al. } \\
(2010)\end{array}$ \\
\hline $\begin{array}{l}\text { Modified Sawdust (cedrus } \\
\text { deodar wood) }\end{array}$ & 73.62 & $\begin{array}{l}\text { Memon et al. } \\
(2007)\end{array}$ \\
\hline Sawdust (Pinus sylvestris) & 9.29 & $\begin{array}{l}\text { Taty-Costodes et } \\
\text { al. (2003) }\end{array}$ \\
\hline Juniper fibre & 29.54 & Min et al. (2004) \\
\hline $\begin{array}{l}\text { Azolla filiculoides (aquatic } \\
\text { fern) }\end{array}$ & 86 & $\begin{array}{l}\text { Ganji et al. } \\
(2005)\end{array}$ \\
\hline
\end{tabular}

Copyright (c) 2018 Authors. This is an open access article distributed under the Creative Commons Attribution License, which permits unrestricted use, distribution, and reproduction in any medium, provided the original work is properly cited. 


\begin{tabular}{|l|c|l|}
\hline $\begin{array}{l}\text { Palm Sheath(Petiolar felt- } \\
\text { sheath) }\end{array}$ & 10.08 & $\begin{array}{l}\text { Iqbal M et } \\
\text { al.(2002) }\end{array}$ \\
\hline Walnut sawdust & 4.51 & $\begin{array}{l}\text { Bulut and Tez } \\
(2003)\end{array}$ \\
\hline Cassava tuber bark waste & 26.3 & $\begin{array}{l}\text { Horsfall Jr. et al. } \\
(2006)\end{array}$ \\
\hline $\begin{array}{l}\text { Cassava Waste(thioglycolic } \\
\text { acid modified) }\end{array}$ & 18.05 & $\begin{array}{l}\text { Abia A.A et } \\
\text { al.(2003) }\end{array}$ \\
\hline Cocoa Shell & 4.94 & $\begin{array}{l}\text { Meunier et } \\
\text { al.(2003) }\end{array}$ \\
\hline Wheat bran & 101 & $\begin{array}{l}\text { Ozer and Pirinc } \\
(2006)\end{array}$ \\
\hline Spent grain & 17.3 & Low et al. (2000) \\
\hline Black oak bark & 25.9 & Masri et al., 1974 \\
\hline Hardwickia binata bark & 34 & $\begin{array}{l}\text { Deshkar } \text { et al., } \\
1990\end{array}$ \\
\hline Redwood bark & 32 & $\begin{array}{l}\text { Masri } \text { et al., } \\
1974 ; \text { Randall } \text { et } \\
\text { al., 1974a,b }\end{array}$ \\
\hline Coirpith activated carbon & 93.4 & $\begin{array}{l}\text { Kadirvelu et al. } \\
(2003)\end{array}$ \\
\hline
\end{tabular}

\subsubsection{Agricultural Products And By-Products}

The biosorption properties sunflower stalk showed an adsorption of $70 \mathrm{mg} / \mathrm{g}$ (Jalali and Aboulghazi 2013), hazelnut husk carbon $(61.35 \mathrm{mg} / \mathrm{g})$ and Annona squamosa $(71 \mathrm{mg} / \mathrm{g})$ (Isaac and Sivakumar 2013). Studies reveal the various fruit peels like jack fruit peel (Inbaraj \& Sulochana 2004), modified orange peel (Feng et al., 2011) and mango peel (M. Iqbal et al., 2009) showed good adsorption and were presented in table 2.

Table 2: Adsorption capacity (mg/g) of agricultural products and byproducts

\begin{tabular}{|c|c|c|}
\hline Adsorbent & $\begin{array}{c}\text { Adsorption } \\
\text { capacity (mg/g) }\end{array}$ & References \\
\hline $\begin{array}{l}\text { Corn stalk } \\
\text { (acrylonitrile } \\
\text { modified) }\end{array}$ & 12.73 & $\begin{array}{l}\text { Zheng et al. (2010a, } \\
\text { 2010b) }\end{array}$ \\
\hline Corn stalk (raw) & 3.39 & $\begin{array}{c}\text { Zheng et al. (2010a, } \\
2010 \mathrm{~b})\end{array}$ \\
\hline Corncobs & 8.89 & $\begin{array}{c}\text { Vaughan T et } \\
\text { al.(2001) }\end{array}$ \\
\hline Corn Starch & 8.88 & Zacaria R (2002) \\
\hline Sunflower stalk & 70 & $\begin{array}{c}\text { Jalali and Aboulghazi } \\
(2013)\end{array}$ \\
\hline $\begin{array}{l}\text { Coffee Residues } \\
\text { (Pyrolyzed) }\end{array}$ & 39.5 & $\begin{array}{c}\text { Boonamuayvitaya } \mathrm{V} \\
\text { et al.(2004) }\end{array}$ \\
\hline Blend coffee & 2.0 & $\begin{array}{l}\text { M. Minamisawa et al. } \\
(2005)\end{array}$ \\
\hline $\begin{array}{l}\text { Modified Orange peel } \\
\text { (OPAA) }\end{array}$ & 293.3 & Feng et al. (2011) \\
\hline Jack fruit Peel & 52.08 & $\begin{array}{c}\text { Inbaraj \& Sulochana } \\
(2004)\end{array}$ \\
\hline Peanut hulls & 5.96 & Brown P et al.(2000) \\
\hline Peanut hulls pellets & 6.0 & Brown P et al.(2000) \\
\hline Hazelnut husk carbon & 61.35 & $\begin{array}{c}\text { Imamoglu et al. } \\
(2014)\end{array}$ \\
\hline Agave bagasee( raw) & 14 & $\begin{array}{c}\text { Velazquez-Jimenez et } \\
\text { al. (2013) }\end{array}$ \\
\hline $\begin{array}{l}\text { Agave sisalana (sisal } \\
\text { fiber) }\end{array}$ & 1.85 & $\begin{array}{c}\text { dos Santos et al. } \\
(2011)\end{array}$ \\
\hline Annona squamosa & 71.0 & $\begin{array}{l}\text { Isaac and Sivakumar } \\
(2013)\end{array}$ \\
\hline Wheat straw & 39.22 & Farooq et al. (2011) \\
\hline Wheat bran & 0.70 & Singh KK et al.(2006) \\
\hline Walnut shell & 1.5 & $\begin{array}{l}\text { Orhan and BuÈ yuÈ } \\
\text { kguÈ ngoÈ r, } 1993\end{array}$ \\
\hline $\begin{array}{l}\text { Functionalized } \\
\text { coconut fibers }\end{array}$ & $0.2-5 \mathrm{mmol} / \mathrm{g}$ & $\begin{array}{l}\text { D.A. de Sousa et al. } \\
(2010)\end{array}$ \\
\hline Coconut copra meal & 4.99 & Y.-S. Ho et al. (2006) \\
\hline $\begin{array}{l}\text { EDTA-modified } \\
\text { groundnut husk }\end{array}$ & $0.36 \mathrm{mmol} / \mathrm{g}$ & $\begin{array}{l}\text { F.E. Okieimen et al. } \\
\text { (1991) }\end{array}$ \\
\hline Banana peel & 35.52 & $\begin{array}{l}\text { J.R.Memon et al. } \\
(2008)\end{array}$ \\
\hline
\end{tabular}

\begin{tabular}{|l|l|l|}
\hline Pomelo peel & 21.83 & M. Iqbal et al.(2009) \\
\hline Mango peel & 68.92 & \\
\hline
\end{tabular}

\subsubsection{Industrial Wastes}

Many industrial wastes are proved to have adsorption capacity towards heavy metal ions. Industrial by-products such as maize cope and husk (Igwe et al., 2005), blast furnace slag (V.K. Gupta et al., 1997), sugarcane baggasse (modified) (Junior $e t$ al., 2006) and sugarcane baggasse carbon (Mohan and Singh, 2002) have maximum capacities in removing toxic cadmium ions from aqueous solutions were given in table 3 .

Table 3: Adsorption capacity ( $\mathrm{mg} / \mathrm{g}$ ) of industrial wastes

\begin{tabular}{|c|c|c|}
\hline Table 3: Adsorption capacity (mg/g) of industrial wastes \\
\hline Adsorbent & $\begin{array}{c}\text { Adsorption } \\
\text { capacity } \mathbf{( m g} / \mathbf{g})\end{array}$ & References \\
\hline Black gram husk & $19.56-49.97$ & A. Saeed et al.(2005) \\
\hline Maize cope and husk & 493.7 & Igwe et al. (2005) \\
\hline Blast furnace slag & 18.72 & V.K. Gupta et al.(1997) \\
\hline Blast furnace sludge & $6.74-10.15$ & $\begin{array}{c}\text { A. Lopez-Delgado et } \\
\text { al(1998) }\end{array}$ \\
\hline Red mud & 10.57 & E. Lopez et al.(1998) \\
\hline $\begin{array}{c}\text { Rice husk(sodium } \\
\text { hydroxide) }\end{array}$ & 20.24 & $\begin{array}{c}\text { Kumar and } \\
\text { Bandyopadhyay (2006) }\end{array}$ \\
\hline $\begin{array}{c}\text { Rice husk(Water } \\
\text { washed) }\end{array}$ & 8.58 & $\begin{array}{c}\text { Kumar and } \\
\text { Bandyopadhyay (2006) }\end{array}$ \\
\hline $\begin{array}{c}\text { Rice husk(sodium } \\
\text { bicarbonate) }\end{array}$ & 16.18 & $\begin{array}{c}\text { Kumar and } \\
\text { Bandyopadhyay (2006) }\end{array}$ \\
\hline $\begin{array}{c}\text { Rice } \\
\text { husk(Epichlorohydrin) }\end{array}$ & 11.12 & $\begin{array}{c}\text { Kumar and } \\
\text { Bandyopadhyay (2006) }\end{array}$ \\
\hline $\begin{array}{c}\text { Wignin-Able slurry } \\
\text { waste of paper \& pulp } \\
\text { industry }\end{array}$ & 15.73 & S.M. Lee et al.(2001) \\
\hline Coffee husk & 12.05 & Demirbas A (2004) \\
\hline Turkish tea waste & $2.59-11.29$ & Oliveira et al. (2008) \\
\hline Tea wastes & 11.3 & Cay S et al.(2004) \\
\hline $\begin{array}{c}\text { Tea wastes(binary } \\
\text { system) }\end{array}$ & 2.59 & Cay S et al.(2004) \\
\hline Sugar beet Pulp & 17.2 & Zacaria R et al.(2002) \\
\hline $\begin{array}{c}\text { Sugarcane } \\
\text { bagasse(Sodium } \\
\text { bicarbonate) }\end{array}$ & 189 & Junior et al. (2006) \\
\hline $\begin{array}{c}\text { Sugarcane bagasse } \\
\text { pith }\end{array}$ & 24.7 & $\begin{array}{c}\text { Krishnan KA \& } \\
\text { Anirudhan TS (2003) }\end{array}$ \\
\hline $\begin{array}{c}\text { Sugarcane Bagasse } \\
\text { carbon }\end{array}$ & 38.03 & Mohand Singh \\
& $2002)$ \\
\hline $\begin{array}{c}\text { Grape bagasse } \\
\text { Al(2004) }\end{array}$ \\
\hline
\end{tabular}

\section{Conclusions}

Adsorption contributes significantly to the remediation of many heavy metals, dyes, organics, phenols etc. Studies conducted on natural materials were reviewed show that plant based biomass Moringa oleifera $(171.37 \mathrm{mg} / \mathrm{g})$, Acacia leucocephala bark powder $(167.7 \mathrm{mg} / \mathrm{g})$, Wheat $\operatorname{bran}(101 \mathrm{mg} / \mathrm{g})$ were having maximum adsorption capacities. Maximum adsorption capacities of agricultural by-products reported were modified orange peel $(293.3 \mathrm{mg} / \mathrm{g})$, annona squamosa $(71 \mathrm{mg} / \mathrm{g})$, sunflower stalk $(70 \mathrm{mg} / \mathrm{g})$. Maize cope and husk $(493.7 \mathrm{mg} / \mathrm{g})$ and Sugarcane bagasse(Sodium bicarbonate) $(189 \mathrm{mg} / \mathrm{g})$ were having maximum adsorption under industrial wastes. This review concludes that adsorption is the best possible option for hassle free remediation of contaminated waters.

\section{References}

[1] Abia A.A, Horsfall Jr., M., Didi, O., 2003. The use of chemically modified and unmodified cassava waste for the 
removal of $\mathrm{Cd}, \mathrm{Cu}$ and $\mathrm{Zn}$ ions from aqueous solution. Bioresour. Technol. 90, 345-348.

[2] Al-Dujaili AH, Awwad AM, Salem NM (2012) Biosorption of cadmium (II) onto loquat leaves (Eriobotrya japonica) and their ash from aqueous solution, equilibrium, kinetics, and thermodynamic studies. International Journal of Industrial Chemistry 3:22

[3] Al-Rashdi B.A.M., D.J. Johnson, N. Hilal, Removal of heavy metal ions by nanofiltration, Desalination 315 (2013) 2-17.

[4] Boonamuayvitaya V, Chaiya C, Tanthapanicchakoon W \& Jarudilokul S, Removal of heavy metals by adsorbent prepared from pyrolyzed coffee residues and clay, Sep Purif Technol,35 (2004) 11-22.

[5] Brown P, I.A. Jefcoat, D. Parrish, S. Gill, S. Graham, Evaluation of the adsorptive capacity of peanut hull pellets for heavy metals in solution, Adv.Environ. Res. 4 (2000) 19-29.

[6] Bulut, Y., Tez, Z., 2003. Removal of heavy metal ions by modified sawdust of walnut. Fresen. Environ. Bull. 12, 14991504.

[7] Cay S, A. Uyanık, A. Ozas, 1k, Single and binary component adsorption of copper(II) and cadmium(II) from aqueous solutions using tea-industry waste, Sep. Purif. Technol. 38 (2004) 273-280

[8] De Sousa D.A., E. de Oliveira, M. da, C. Nogueira, B.P. Esposito, Development of a heavy metal sorption system through the P S functionalization, of coconut (Cocos nucifera) fibers, Bioresour. Technol. 101 (2010) 138-143.

[9] Demirbas A (2004) Adsorption of lead and cadmium ions in aqueous solutions into modified lignin from alkali glucerol delignification. J of hazard Mater, 109: 221-226.

[10] Deshkar AM, Bokade SS, Dara SS (1990). Modified Hardwickia Binata bark for adsorption of mercury (II) from water. Water Res. 24(8):1011-1016.

[11] Dos Santos WNL, Cavalcante DD, da Silva EGP, CF d V, FS D (2011) Biosorption of $\mathrm{Pb}(\mathrm{II})$ and $\mathrm{Cd}(\mathrm{II})$ ions by Agave sisalana (sisal fiber). Microchem J 97:269-273

[12] Farinella N.V, G.D. Matos, E.L. Lehmann, M.A.Z. Arruda, Grape bagasse as an alternative natural adsorbent of cadmium and lead for effluent treatment, J.Hazard. Mater. 154 (2004) 1007-1012.

[13] Farooq U, Khan MA, Atharc M, Kozinskia JA (2011) Effect of modification of environmentally friendly Bioadsorbents wheat (Triticum aestivum) on the biosorptive removal of cadmium(II) ions from aqueous solution. Chem Eng J 171:400-410

[14] Feng N, Guo X, Liang S, Zhu Y, Liu J (2011) Biosorption of heavy metals from aqueous solutions by chemically modified orange peel. J Hazard Mater 185:49-54

[15] Ganji, M.T., Khosravi, M., Rakhsaee, R., 2005. Biosorption of $\mathrm{Pb}, \mathrm{Cd}, \mathrm{Cu}$ and $\mathrm{Zn}$ from wastewater by treated Azolla filiculoides with $\mathrm{H} 2 \mathrm{O} 2 / \mathrm{MgCl} 2$. Int. J. Environ. Sci. Technol. 1, 265-271.

[16] Gupta V.K, A. Rastogi, M.K. Dwivedi, D. Mohan, Process development for the removal of zinc and cadmium from wastewater using slag - a blast-furnace waste material, Sep. Sci. Technol. 32 (1997) 2883-2912.

[17] Ho Y.S, A.E. Ofomaja, Biosorption thermodynamics of cadmium on coconut copra meal as biosorbent, Biochem. Eng. J. 30 (2006) 117-123.

[18] Horsfall Jr., M., Abia, A.A., 2003. Sorption of cadmium(II) and zinc(II) ions from aqueous solutions by cassava waste biomass (Manihot sculenta Cranz). Water Res. 37, 4913-492.

[19] Igwe J. C, D. N. Ogunewe, and A. A. Abia. "Competitive adsorption of $\mathrm{Zn}$ (II), $\mathrm{Cd}$ (II) and $\mathrm{Pb}$ (II) ions from aqueous and non-aqueous solution by maize cob and husk." African Journal of Biotechnology 4.10 (2005): 1113.

[20] Imamoglu M, Yildiz H, Altundag H, Turhan Y (2014) Efficient Removal of Cd(II) from Aqueous Solution by Dehydrated Hazelnut Husk Carbon. J Dispersion Sci Technol 36(2):284290

[21] Inbaraj, B.S., Sulochana, N., 2004. Carbonised jackfruit peel as an adsorbent for the removal of $\mathrm{Cd}(\mathrm{II})$ from aqueous solution. Bioresource Technology 94, 49-52.

[22] Iqbal M, Saeed A, Akhtar N (2002) Petiolar felt sheath of palm: a new adsorbent for the removal of heavy metals from contaminated water. Bioresour Technol 81:151-153.

[23] Iqbal M., A. Saeed, S.I. Zafar, FTIR spectrophotometry, kinetics and adsorption isotherms modeling, ion exchange, and EDX analysis for understanding the mechanism of $\mathrm{Cd} 2+$ and $\mathrm{Pb} 2+$ removal by mango peel waste, J. Hazard. Mater.164 (2009) $161-171$.

[24] Isaac CPJ, Sivakumar A (2013) Removal of lead and cadmium ions from water using Annona squamosa shell: kinetic and equilibrium studies. Desalina Water Treat 51:7700-7709J. 157 (2010) 238-248.

[25] Jalali M, Aboulghazi F (2013) Sunflower stalk, an agricultural waste, as an adsorbent for the removal of lead and cadmium from aqueous solutions. J Mater Cycles Waste Manag 15:548555

[26] Junior O.K, Gurgel, L.V.A., de Melo, J.C.P., Botaro, V.R., Melo, T.M.S., de Freitas Gil, R.P., Gil, L.F., 2006. Adsorption of heavy metal ion from aqueous single metal solution by chemically modified sugarcane bagasse. Bioresour. Technol. 98 , 1291-1297.

[27] Kadirvelu, K., Kavipriya, M., Karthika, C.,Radhika, M., Vennilamani, N., and Pattabhi S, (2003). Utilization of Various Agricultural Wastes for Activated Carbon Preparation and Application for the removal of dyes and metal ions from Aqueous Solution. Bioresource Tech. 87: 129-132 pp.

[28] Kang S.Y, J.U. Lee, S.H. Moon, K.W. Kim, Competitive adsorption characteristics of $\mathrm{Co} 2+, \mathrm{Ni}+2$, and $\mathrm{Cr} 3+$ by IRN-77 cation exchange resin in synthesized wastewater, Chemosphere 56 (2004) 141-147.

[29] Khosravi J, A. Alamdari, Copper removal from oil-field brine by coprecipitation, J. Hazard. Mater. 166 (2009) 695-700.

[30] Krishnan KA, Anirudhan TS (2003) Removal of cadmium(II) from aqueous solutions by steamactivated sulphurised carbon prepared from sugar-cane bagasse pith: kinetic and equilibrium studies. Water SA 29(2):147-156.

[31] Kumar U, M. Bandyopadhyay, Sorption of cadmium from aqueous solution using pretreated rice husk, Bioresour. Technol. 97 (2006) 104-109.

[32] Lee S. M and A. P. Davis, Removal of Cu(II) and Cd(II) from aqueous solution by seafood processing waste sludge, Water Res., 2001, 35(2), 534-540.

[33] Liu Y, Chang X, Guo Y \& Meng S, Biosorption and preconcentration of lead and cadmium on Chinese waste herb Pang Da Hai, J Hazard Master,135(2006) 389-394.

[34] Lopez E, B. Soto, M. Arias, A. Nun' ez, D. Rubinos, M.T. Barral, Adsorbent properties of red mud and its use for wastewater treatment, Water Res. 32 (1998) 1314-1322.

[35] Lopez-Delgado.A, C. Perez, F.A. Lopez, Sorption of heavy metals on blast furnace sludge, Water Res. 32 (1998) 989-996.

[36] Low K.S, Lee, C.K., Liew, S.C., 2000. Sorption of cadmium and lead from aqueous solutions by spent grain. Process Biochem. 36, 59-64.

[37] Masri, M.S., Reuter, F.W., Friedman, M., 1974. Binding of metal cations by natural substances. J. Appl. Polym. Sci. 18, 675-681.

[38] Mauri R, R. Shinnar, M.D. Amore, P. Giordano, A. Volpe, Solvent extraction of chromium and cadmium from contaminated soils, AIChE J. 47 (2001) 509-512.

[39] Memon J.R, S.Q.Memon,M.I. Bhanger, G. ZuhraMemon,A. ElTurki, G.C. Allen, Characterization of banana peel by scanning electron microscopy and FT-IR spectroscopy and its use for cadmium removal, Colloids Surf., B: Biointerfaces 66 (2008) 260-265.

[40] Memon, S.Q., Memon, N., Shah, S.W., Khuhawar, M.Y., Bhanger, M.I., 2007. Sawdust - a green and economical sorbent for the removal of cadmium(II) ions. J. Hazard. Mater. B 139, 116-121.

[41] Meunier N, Laroulandie J, Blais JF, Tyagi RD (2003) Cocoa shells for heavy metal removal from acidic solutions. Bioresour Technol 90:255-263

[42] Min S.H., Han, J.S., Shin, E.W., Park, J.K., 2004. Improvement of cadmium ion removal by base treated juniper fiber. Water Res. 38, 1289-1295.

[43] Minamisawa M, S. Nakajima, H. Minamisawa, S. Yoshida, N. Takai, Removal of copper (II) and cadmium(II) from water using roasted coffee beans, in: Eric Lichtfouse, Jan Schwarzbauer, Didier Robert (Eds.), Environmental Chemistry Green Chemistry and Pollutants in Ecosystems, Springer, Berlin Heidelberg, 2005, pp. 259-265.

[44] Mohammadi T, A. Mohebb, M. Sadrzadeh, A. Razmi, Modeling of metal ion removal from wastewater by electrodialysis, Sep. Purif. Technol. 41 (2005) 73-82. 
[45] Mohan D, Singh KP (2002) Single- and multi-component adsorption of cadmium and zinc using activated carbon derived from bagasse-an agricultural waste. Water Res 36:2304-2318

[46] Munagapati VS, Yarramuthi V, Nadavala SK (2010) Biosorption of $\mathrm{Cu}(\mathrm{II}), \mathrm{Cd}(\mathrm{II})$ and $\mathrm{Pb}(\mathrm{II})$ by Acacia leucocephala bark powder: Kinetics, equilibrium and thermodynamics. Chem Eng J 157:357-365.

[47] Ozer, A., Pirinc c ci, H.B., 2006. The adsorption of $\mathrm{Cd}(\mathrm{II})$ ions on sulfuric acid-treated wheat bran. J. Hazard. Mater. B 137, 849-855.

[48] Okieimen F.E., E.U. Okundia, D.E. Ogbeifun, Sorption of cadmium and lead ions on modified groundnut (Arachis hypogea) husks, J. Chem. Technol. Biotechnol.51 (1991) 97103.

[49] Oliveira WE, Franca AS, Oliveira LS, Rocha SD (2008) Untreated coffee husks as biosorbents for the removal of heavy metals from aqueous solutions. J Hazard Mater. 152: 1073-81.

[50] Purkayastha D, Mishra U, Biswas S (2014) A comprehensive review on $\mathrm{Cd}$ (II) removal from aqueous solution Journal of Water Process Engineering 2:105-128

[51] Randall JM, Bermann RL, Garrett Vand, Waiss AC Jr. (1974a). Use of bark to remove heavy metal ionsfrom waste solutions. For. Prod. J.24(9): 80-84. Randall JM, Hautala E, Waiss AC Jr. (1974b). Removal and recycling of heavy metal ions from mining and industrial waste streams with agricultural byproducts. Proceedings of the Fourth Mineral Waste Utilization Symposium. Chicago, IL, May 1974. pp. 329-334.

[52] Rao RAK, Rehman F (2012) Use of Polyalthia longifolia Seeds (Seeds of Indian Mast Tree) as Adsorbent for the removal of Cd(II) from Aqueous Solution. J Dispersion Sci Technol 33:472-48.

[53] Reddy DHK, Seshaiah K, Reddy AVR, Lee SM (2012) Optimization of $\mathrm{Cd}(\mathrm{II}), \mathrm{Cu}(\mathrm{II})$ and $\mathrm{Ni}(\mathrm{II})$ biosorption by chemically modified Moringa oleifera leaves powder Carbohydr Polym 88:1077-1086.

[54] Rubio J, M.L. Souza, R.W. Smith, Overview of flotation as a wastewater treatment technique, Miner. Eng. 15 (2002) 139155.

[55] Saeed A., M. Iqbal, W. Akhtar, Removal and recovery of lead(II) from single and multimetal $(\mathrm{Cd}, \mathrm{Cu}, \mathrm{Ni}, \mathrm{Zn})$ solutions by crop milling waste (black gram husk), J. Hazard. Mater. 117 (2005) 65-73.

[56] Saikaew W, P. Kaewsarn, W. Saikaew, Pomelo Peel: agricultural waste for biosorption of cadmium ions from aqueous solutions, World Acad. Sci. Eng. Technol. 56 (2009) 287-291.

[57] Shameem, H., Abburi, K., Tushar, K. G., Dabir, S. V., Veera, M. B., \& Edgar, D. S. (2006), Adsorption of divalent cadmium (Cd(II)) from aqueous solutions onto chitosancoated perlite beads. Industrial \& Engineering Chemistry Research, 45, 50665077.

[58] Singh K.K, A.K. Singh, S.H. Hasan, Low cost bio-sorbent 'wheat bran' for the removal of cadmium from wastewater: kinetic and equilibrium studies, Bioresour. Technol. 97 (2006) 994-1001.

[59] Taty-Costodes, V.C., Favdvet, H., Porte, C., Delacroix, A., 2003. Removal of cadmium and lead ions from aqueous solutions, by adsorption onto saw dust of Pinus sylvestris. J. Hazard. Mater. B105, 121-142.

[60] Vaughan, T., Seo, C.W., Marshall, W.E., 2001. Removal of selected metal ions from aqueous solution using modified corncobs. Bioresource Technology 78 (2), 133-139.

[61] Velazquez-Jimenez LH, Pavlick A, Rangel-Mendez JR (2013) Chemical characterization of raw and treated agave bagasse and its potential as adsorbent of metal cations from water. Ind Crop Prod 43:200-206

[62] Viraraghavan T, Dronamrajum (1993). Removal of copper, nickel and zinc from wastewater by adsorption using peat. J. Environ. Sci. Health Part A 28: 1261.

[63] Zacaria R (2002). Adsorption of several metal ions onto lowcost biosorbent: kinetic and equilibrium studies. Environ. Sci. Technol. 36:2067-2073.

[64] Zheng L, Dang Z, Yi X, Zhang H (2010a) Equilibrium and kinetic studies of adsorption of $\mathrm{Cd}(\mathrm{II})$ from aqueous solution using modified corn stalk. J Hazard Mater 176:650-656. Zheng L, Dang Z, Yi X, Zhang H (2010b) Removal of cadmium (II) from aqueous solution by corn stalk graft copolymers. Bioresour Technol 101:5820-5826. 